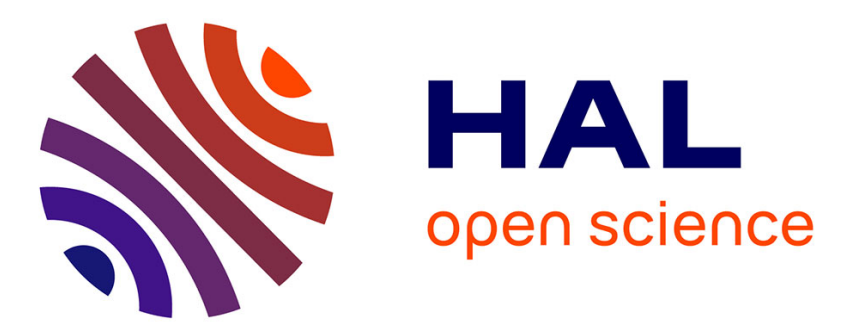

\title{
VScope: Middleware for Troubleshooting Time-Sensitive Data Center Applications
}

Chengwei Wang, Infantdani Abel Rayan, Greg Eisenhauer, Karsten Schwan, Vanish Talwar, Matthew Wolf, Chad Huneycutt

\section{To cite this version:}

Chengwei Wang, Infantdani Abel Rayan, Greg Eisenhauer, Karsten Schwan, Vanish Talwar, et al.. VScope: Middleware for Troubleshooting Time-Sensitive Data Center Applications. 13th International Middleware Conference (MIDDLEWARE), Dec 2012, Montreal, QC, Canada. pp.121-141, 10.1007/978-3-642-35170-9_7 . hal-01555552

\section{HAL Id: hal-01555552 \\ https://hal.inria.fr/hal-01555552}

Submitted on 4 Jul 2017

HAL is a multi-disciplinary open access archive for the deposit and dissemination of scientific research documents, whether they are published or not. The documents may come from teaching and research institutions in France or abroad, or from public or private research centers.
L'archive ouverte pluridisciplinaire HAL, est destinée au dépôt et à la diffusion de documents scientifiques de niveau recherche, publiés ou non, émanant des établissements d'enseignement et de recherche français ou étrangers, des laboratoires publics ou privés.

\section{(c)(1)}

Distributed under a Creative Commons Attribution| 4.0 International License 


\title{
VScope: Middleware for Troubleshooting Time-Sensitive Data Center Applications
}

\author{
Chengwei Wang, Infantdani Abel Rayan ${ }^{+}$, Greg Eisenhauer, \\ Karsten Schwan, Vanish Talwar*, Matthew Wolf, Chad Huneycutt \\ Georgia Institute of Technology ${ }^{*} \mathrm{HP}$ Labs ${ }^{+}$Riot Games
}

\begin{abstract}
Data-Intensive infrastructures are increasingly used for online processing of live data to guide operations and decision making. VScope is a flexible monitoring and analysis middleware for troubleshooting such large-scale, time-sensitive, multi-tier applications. With VScope, lightweight anomaly detection and interaction tracking methods can be run continuously throughout an application's execution. The runtime events generated by these methods can then initiate more detailed and heavier weight analyses which are dynamically deployed in the places where they may be most likely fruitful for root cause diagnosis and mitigation. We comprehensively evaluate VScope prototype in a virtualized data center environment with over 1000 virtual machines (VMs), and apply VScope to a representative on-line log processing application. Experimental results show that VScope can deploy and operate a variety of on-line analytics functions and metrics with a few seconds at large scale. Compared to traditional logging approaches, VScope based troubleshooting has substantially lower perturbation and generates much smaller log data volumes. It can also resolve complex cross-tier or cross-software-level issues unsolvable solely by application-level or per-tier mechanisms.
\end{abstract}

Keywords: Cloud, Data Center, Management, Troubleshooting

\section{Introduction}

In the 'big data' era, live data analysis applications are becoming easy to scale, as well as being lucrative for or even critical to a company's operation. For instance, by continuously analyzing the live number of page views on its products, an ecommerce website can run a dynamic micro-promotion strategy in which when over 3000 customers are looking at a product for over 10 seconds, an extra $20 \%$ discount appears on the web page to increase sales. Other mission-critical examples for e-commerce sites are click fraud and spam detection.

The importance of live data analysis is underscored by the recent creation of real-time or 'streaming' big data infrastructures ${ }^{1}$, which include Flume, S4, Storm, Chukwa, and others $[5,28,25,29,11,23,15]$. Conceptually, these are based

\footnotetext{
${ }^{1}$ In this paper we use the term 'real-time' to refer a latency restriction within seconds or hundreds of milliseconds.
} 


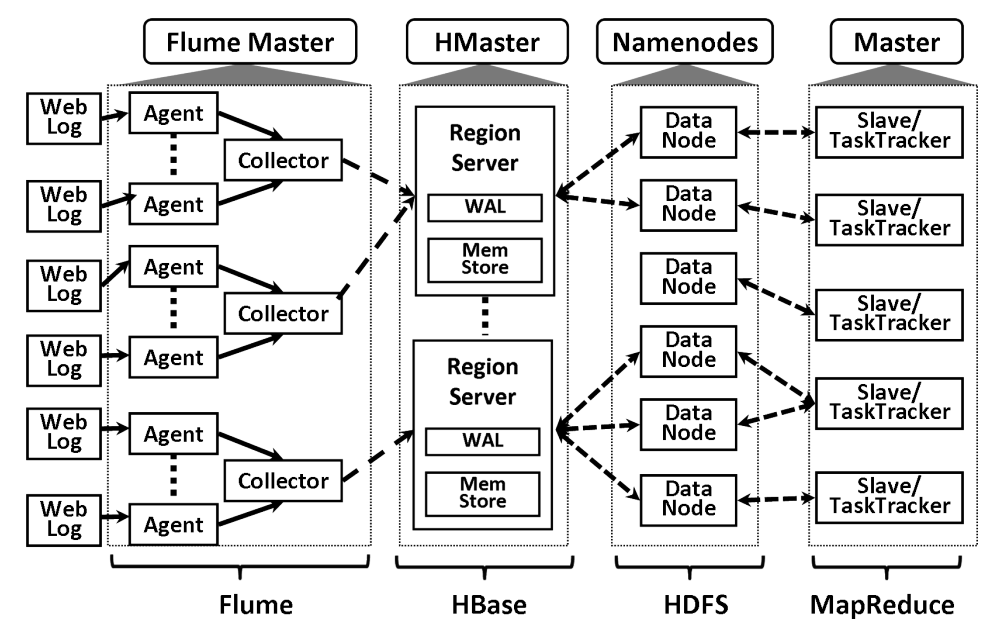

Fig. 1. A typical real-time web log analysis application composed from Flume, HBase, HDFS, and Hadoop. In Flume, agents reside in web or application servers, collecting logs and converting them into key-value pairs. Collectors receive and aggregate the local results and insert them into HBase, a distributed, scalable key-value store by which users can query the analysis results on-the-fly. HBase consists of region servers that are equipped with a memory cache, termed MemStore, and a Write Ahead Log (WAL). The data are first written to the WAL and MemStore before being asynchronously persisted to the back-end distributed file system, HDFS, which is typically shared by other data-intensive batch systems, such as Hadoop-based MapReduce codes used for off-line, long-term analyses. Each tier can scale to 1000s of servers or virtual machines.

on the well-established paradigm of stream- or event-based processing $[16,2,1]$, but their attractiveness stems from the fact that they can be easily integrated with other elements of 'big data' infrastructures, such as scalable key-value stores and MapReduce systems, to construct multi-tier platforms spanning thousands of servers or consolidated virtual servers in data centers. A sample platform integrating Flume and other data-intensive systems is depicted in Figure 1.

Crucial to maintaining high availability and performance for these multi-tier applications, particularly in light of their stringent end-to-end timing requirements, is responsive troubleshooting - a process involving the timely detection and diagnosis of performance issues. Such troubleshooting is notoriously difficult, however, for the following reasons:

- Holistic vs. tier-specific troubleshooting. As illustrated in Figure 1, each tier is typically a complex distributed system with its own management component, e.g. HBase or Flume masters. Developed by different vendors and/or managed by different operation teams, tier-specific management can improve the availability of individual tiers, but is not sufficient for maintaining an entire application's end-to-end performance, a simple reason being that issues visible in one tier may actually be caused by problems located in another. Needed are holistic systems to efficiently track problems across tiers. 
- Dynamic, per-problem functionality. Problems in complex, large-scale systems arise dynamically, and for each class of problems, there may be different detection, analysis, and resolution methods. Troubleshooting, therefore, is an inherently dynamic activity, involving on-line capabilities to capture differing metrics and to diagnose/analyze them with potentially problemand situation-specific methods[36].

- Scalable, responsive problem resolution. In latency-sensitive applications like the one in Figure 1, to maintain desired timing, troubleshooting must be conducted both with low perturbation and with high responsiveness: issues must be detected, diagnosed, and repaired without missing too many events and while maintaining availability for other ongoing actions.

- System-level effects. Holistic troubleshooting must extend beyond a single application, to also identify the system-level bottlenecks that can arise in today's consolidated data center or cloud computing systems.

Previous troubleshooting systems have not addressed all of these challenges. Solutions that monitor 'everything all the time' $[26,39,27]$, including both application and system-level events, do not scale for detailed diagnostics via say, debug-level logging or tracing with consequent high levels of perturbation. This is shown in Figure 2, where continuously logging applicationlevel debugging events on all of its nodes slows down an application's performance by more than 10 times over the baseline. Sampling $[30,31,14,7]$ for some of the components and/or for some period of time may not only miss important events,

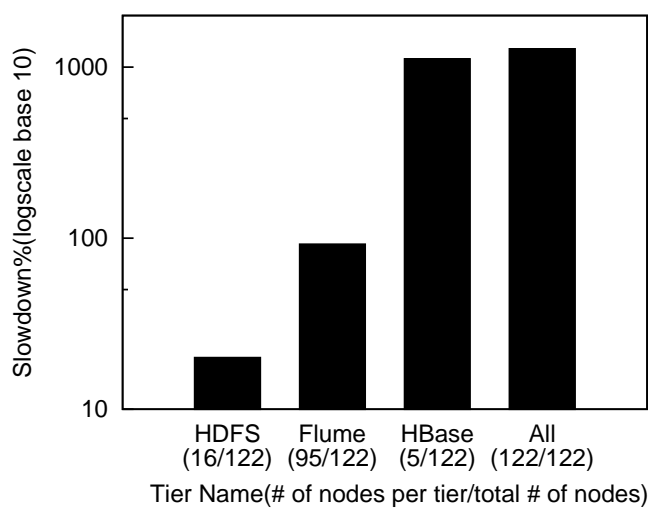

Fig. 2. E2E performance slowdown (i.e. latency increase) \% caused by debug-level logging at difaffecting troubleshooting effec- ferent tiers of the architecture shown in Figure 1.
tiveness, but will also bring about serious performance issues when using a homogeneous and/or random sampling strategy across all nodes, e.g., with Dapper [31]'s use of a uniform, low (1/1000) sampling rate. In Figure 2, debug-level logging in the Flume application's HBase tier, the smallest portion of the system (5/122 VMs), results in over 10 times slowdown, which is more than an order of magnitude of the perturbation imposed by debug-level logging in the Flume tier, which has the majority of nodes $(95 / 122)$. Thus, it is inadvisable to use a high sampling rate for the HBase tier, whereas such a strategy for the Flume tier will likely lead to only modest additional perturbation. An alternative troubleshooting approach chosen by GWP [30] is to randomly pick some set of machines. This may work well if that set is in the HDFS tier, but will be prohibitively costly if the HBase tier is picked. Other approaches, like those taken by Fay [14] and Chopstix [7] to set sampling rates based on the event population, still remain 
unaware of application level perturbation, resulting in the same issue as the one faced by GWP. We, therefore, conclude that a more flexible system is needed for efficient troubleshooting, where methods can differ for each behavior, tier, and type of analysis being performed.

The VScope middleware presented in this paper makes it possible (1) to adjust and tune troubleshooting dynamically - at runtime - for individual tiers and across tiers, (2) to dynamically deploy any analysis action(s) needed to understand the metric data being captured in the ways required by such troubleshooting, and (3) to do so in ways that meet the perturbation/overhead requirements of target applications. To achieve those ends, VScope, as a flexible monitoring and analysis system, offers the following novel abstractions and mechanisms for troubleshooting latency-sensitive, multi-tier data center applications:

1. Dynamic Watch, Scope, and Query. VScope abstracts troubleshooting as a process involving repeated Watch, Scope, and Query operations. Respectively, these (i) detect performance anomalies, (ii) 'zoom-in' to candidate problematic groups of components or nodes, and (iii) answer detailed questions about those components or nodes using dynamically deployed monitoring or analysis functions. VScope can operate on any set of nodes or software components and thus, can be applied within a tier, across multiple tiers, and across different software levels.

2. Guidance. Replacing the current manual 'problem ticket' mechanisms used in industry, VScope based troubleshooting is directed by on-line 'guidance', realized by the Watch and Scope operations that first detect abnormal behavior, followed by exploring candidate sources for such behavior, and only then initiate more detailed queries on select entities. The current implementations of Watch and Scope support both 'horizontal guidance', to track potential problems across different tiers of a multi-tier application, and 'vertical guidance', to understand whether problems are caused by how applications are mapped to underlying machines.

3. Distributed Processing Graphs (DPGs). All VScope operations are realized by DPGs, which are overlay networks capable of being dynamically deployed and reconfigured on any set of machines or processes, supporting various types of topologies and analysis functionalities. First introduced in our previous work [36], where we proposed the basic architecture of DPGs and investigated an impact model of metric number/size and various DPG topologies, along with other factors, this paper presents DPG implementation, APIs, and commands, based on which we build VScope's troubleshooting functionality.

VScope's capabilities and performance are evaluated on a testbed with over 1000 virtual machines (VMs). Experimental results show the VScope runtime negligibly perturbs system and application performance, and requires mere seconds to deploy 1000 node DPGs of varying topologies. This results in fast operation for on-line queries able to use a comprehensive set of application to system/platform level metrics and a variety of representative analytics functions. When supporting algorithms with high computation complexity, VScope serves as a 'thin layer' that occupies no more than $5 \%$ of their total latency. Further, by using guidance 
that correlates system- and application-level metrics, VScope can locate problematic VMs that cannot be found via solely application-level monitoring, and in one of the use cases explored in the paper, it operates with levels of perturbation of over $400 \%$ less than what is seen for brute-force and most sampling-based approaches.

\section{System Design and Implementation}

\subsection{Goals and Non-Goals}

The design of VScope is driven by the following goals: (1) flexibility: to initiate, change, and stop monitoring and analysis on any set of nodes at any time, supported by operators for dynamically building and controlling user-defined actions for runtime troubleshooting; (2) guided operation: programmable methods for detecting potential problems and then tracking interactions that may contribute to them, between tiers and across software levels, thereby focusing troubleshooting in ways that can reduce overheads and improve effectiveness; and (3) responsiveness and scalability: to deploy troubleshooting methods with low delay at scales of $1000+$ nodes.

VScope is designed to be a general platform rather than a set of ad hoc analysis algorithms/solutions. VScope does not replace operator involvement, but aims to facilitate their troubleshooting efforts. Further, while VScope may be used to seek the root causes of failures, its current implementation lacks functionality like an off-line diagnostic database and a rich infrastructure for determining and using decision trees or similar diagnostic techniques. Also, the methods presently implemented in VScope focus on persistent performance problems that will likely render an application inoperable after some time, i.e., when there are frequent or repeated violations of certain performance indicators that persist if they are not addressed. Having determined potential sources of such problems, VScope can then trigger certain actions for mitigation or recovery, but it assumes such functionality to be supported by other subsystems (e.g., inherent to specific applications/tiers or software levels) or housed in some external system for problem resolution [9].

\subsection{VScope Overview}

The system architecture of VScope is depicted in Figure 3(a). The machines (VMs or physical machines) in the target application are managed by a server called VMaster. Operators use VScope operations, DPG commands, or scripts with the DPG API, in a console called VShell provided by VMaster. VMaster executes those commands by deploying DPGs on requested machines to process their monitoring metrics, and it returns results to operators. In detail, it starts a DPGManager to create a new DPG, which essentially, is an overlay network consisting of processing entities named VNodes residing on application machines. The DPGManager dynamically deploys VNodes equipped with assigned functions on specified machines, and connects them with a specified topology. VNodes 


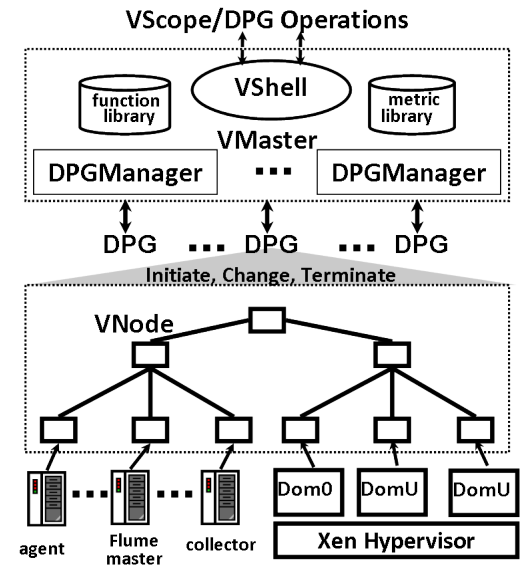

(a) VScope Architecture

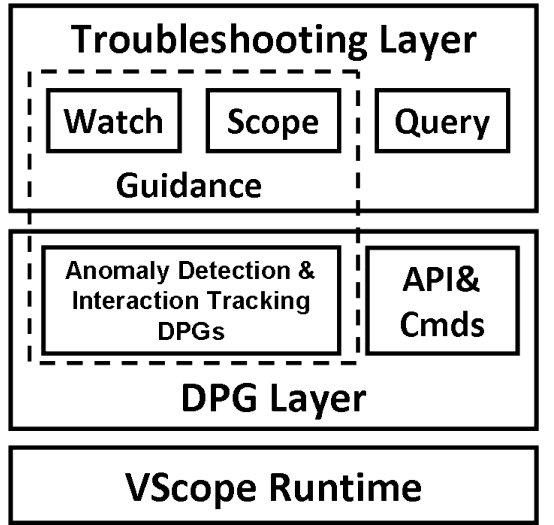

(b) VScope Software Stack

Fig. 3. VScope System Design

collect and process monitoring metrics, transmit metrics or analysis results to other VNodes or the DPGManager, which in turn relays results to VMaster. DPGManager can initiate, change, or terminate its DPG on-the-fly.

In VMaster, the metric library defines monitoring metric types and associated collection functions. The function library defines the user-defined and default metric analysis functions, including those used in guidance (see Section 2.5). The above metrics and functions can be dynamically deployed into DPGs for various troubleshooting purposes.

The VScope software stack, described in Figure 3(b), has three layers. The troubleshooting layer exposes basic operations in VShell: Watch, Scope, and Query, which will be described in Section 2.3. The Watch and Scope operations constitute the guidance mechanism, where Watch notifies the operator when and where end-to-end anomalies happen, and Scope provides the potential candidate nodes contributing to the anomalies. Operators (or automated decision engines) can then use Query for in-depth analysis on those candidates yielded by guidance. These operations are built upon the DPG layer. In particular, the guidance mechanism (Watch and Scope) relies on an anomaly detection DPG and on interaction tracking DPGs. The DPG layer also exposes API and management commands to offer finer grain controls and customization. The lowest layer, the VScope runtime, is comprised of a set of daemon processes running on all nodes participating in the VScope system (i.e., the machines hosting the application and additional management machines running VScope). This runtime maintains the connections between machines and implements dynamic DPG creation and management. In virtualized data centers, the VScope runtime can be installed in hypervisors (e.g., Dom0 in Xen), in the virtual machines hosting the application(s) being monitored, in both, and/or in specialized management engines [21, 24]. Our testbed uses a VScope installation in the Xen hypervisor as well as in the VMs hosting the Flume application. 


\subsection{Troubleshooting Operations}

Watch. The Watch operation monitors a list of metrics on a set of nodes ${ }^{2}$, and its current implementation applies to them an anomaly detection function in order to detect and report anomalous behaviors for any specified metrics. The parameters of the Watch operation described in Table 1 show its ability to monitor metrics on any VScope node, using detection function specified with detectFunc. Sample functions used

Table 1. Arguments of Watch $\left({ }^{*}\right.$ Optional $)$

\begin{tabular}{|c|c|}
\hline Argument & Description \\
\hline \hline nodeList* & a list of nodes to monitor \\
metricList & a list of metric types \\
detectFunc* & detection function or code \\
duration* & duration \\
frequency* & frequency \\
\hline
\end{tabular}

in our work include thresholding key performance indicators (KPI), such as request latency and statistics like those based on entropy described in [37]. The frequency and duration of the Watch operation are also configurable. In our Flume application, the Watch operation continuously executes on all the Flume agent nodes, monitoring their end-to-end message latencies and detecting the nodes with latency outliers. Internally, Watch is implemented using an anomaly detection DPG explained in Section 2.5.

Scope. The Scope operation (described in Table 2) discovers a set of nodes interacting with a particular node specified by argument source, at a time specified by argument timestamp. This operation guides troubleshooting by informing operators which nodes are related to the problematic node when the anomaly happens. Based on this guidance, operators can deploy a DPG on those nodes (or some subset of them) for further diagnosis, using the Query opera-

Table 2. Arguments of Scope $\left(*^{*}\right.$ Optional $)$

\begin{tabular}{|c|c|}
\hline Argument & Description \\
\hline \hline nodeList* & a list of nodes to explore \\
graph & name of interaction graph \\
source & node in interest \\
timestamp* & interaction at a specific time \\
distance & number of edges \\
direction* & backward, forward or both \\
\hline
\end{tabular}
tion. For instance, for the Flume application, 'horizontal guidance' identifies the HBase region servers with which some specified Flume agent is interacting via a Flume collector, and 'vertical guidance' tracks the mappings between a physical machine and the VMs it hosts. By default, the output of Scope is a list of nodes directly interacting with the source. distance and direction are optional arguments, where the former specifies indirect interactions by setting the value $>1$, and the latter specifies the 'direction' of interaction, for instance, 'receiving requests from' or 'sending requests to'.

In a nutshell, Scope works by searching an in-memory, global graph abstraction that describes interactions between every pair of nodes. Multiple types of interaction graphs are supported, covering a range of interactions from event level to network and system levels. These are shown in Table 7 and are specified

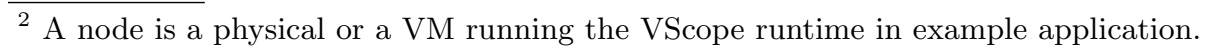


with the argument graph. The creation and continuous update of the global graph is implemented using an interaction tracking DPG explained in Section 2.5.

Query. The Query function collects and analyzes metrics from a specified list of nodes, and provides results to query Table 3. Arguments of Query $\left({ }^{*}\right.$ Optional $)$ initiators. Query has two modes continuous mode and one-shot - the latter being helpful when running monitoring or analysis actions that have high overheads. Query (including query with 'continuous' mode) is de-

\begin{tabular}{|c|c|}
\hline Argument & Description \\
\hline \hline nodeList* & a list of nodes to query \\
metricList* $^{*}$ & a list of metric types \\
queryFunc & analytics function or code \\
mode* $^{*}$ & continuous or one-shot \\
\hline
\end{tabular}
signed with the 'pull' model, i.e., the VMaster requests (pulls) metrics/results from VNodes. Conversely, watch is designed with the 'push' model, i.e., VNodes periodically report basic metrics or anomaly detection results to the VMaster.

\section{$2.4 \quad$ Flexible DPGs}

DPG as the Building Block. All VScope operations described in Section 2.3 are implemented via DPGs. A DPG consists of a set of processing points (VNodes) to collect and analyze monitoring data. It can be configured in multiple topologies to meet varying scale and analysis requirements. For example, it can be configured as a hierarchical tree or as a peer-to-peer overlay or, when operating at smaller scales, as a centralized structure. Managed by a DPGManager, a DPG can be dynamically started on a specified set of nodes, where each VNode runs locally on a designated node and executes functions specified in VScope operations. These functions are stored as binaries in the function library, and they can be dynamically linked. As a result, DPGs are flexible in terms of topology, functions executed, and metric types. Further, DPG outputs can be (i) presented immediately to the VScope user in VShell, (ii) written into rotating logs, or (iii) stored as off-line records in a database or key-value store. The last two configurations are particularly important when historical data is needed for troubleshooting. The use case in Section 4.2 uses rotating logs to store past metric measurements.

DPG API and Management Commands. Figure 4 describes the DPG core API and sample topologies, with details shown in Table 4 . The create() method automatically creates any size topology of type point-to-point $(\mathrm{P})$, centralized $(\mathrm{C})$, or hierarchy $(\mathrm{H})$ for some specified list of nodes. Topology specifics are configurable, e.g., besides the number of nodes, one can specify

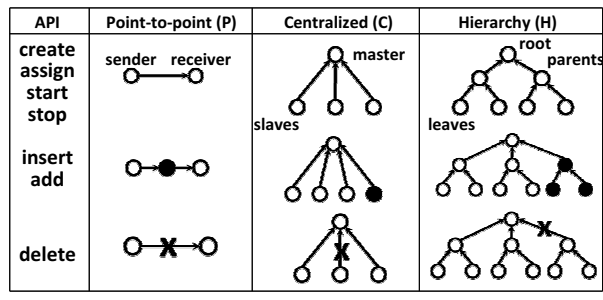

Fig. 4. DPG API and Topologies the branching factor of a hierarchical topology. Create() returns a unique DPG 
Table 4. Pseudo Functions for DPG API

\begin{tabular}{|l|l|}
\hline DPG create (list, topology, spec) & Create a DPG with a specified topology \\
int add (src, dst, DPG) & Add a link from VNode src to VNode dst \\
int assign (func, spec, list, DPG) & Assign function to a list of VNodes \\
int start (DPG) & Start a DPG \\
int stop (DPG) & Stop an operating DPG \\
int insert (new, src, dst, DPG) & Insert a new VNode between existing VNodes \\
int delete (src, dst, DPG) & Delete a link from VNode src to VNode dst \\
\hline
\end{tabular}

ID for reference in subsequent operations, and in the assign() method, the parameter func is a registered function ID. When a DPG is running, one can call the assign() to change the functionality on any VNode or use the insert() and delete() methods to change the DPG. The DPG API is exposed as commands in $V S h e l l$, as well, and there are additional auxiliary management commands like list (listing metric types, functions, or DPGs) and collect (returns the metric collection function).

Though operators can just use VScope operations without knowing the underlying DPG logic, new topologies, new operations and customization of existing functionality can be added easily through direct use of DPG APIs, which is not described in detail here because of space constraints.

\subsection{Implementation}

VScope Runtime. The VScope runtime is implemented with EVPath [1], a C library for building active overlay networks. Metric collection uses standard C libraries, system calls, and JMX (at application level). Metrics are encoded in an efficient binary data format [1], and a standard format template is used to define new metric types. Built-in metrics and functions are listed in Table 5 and Table 6 . As shown in the tables, VScope has a comprehensive set of metrics across application, system and platform levels, and a variety of representative analytics functions that are implemented with standard $\mathrm{C}$ libraries and other open source codes [13]. The DPGs associated with these functions have different topologies. For instance, Pathmap, PCA (Principle Component Analysis) and $\mathrm{K}$-Clustering are implemented as centralized DPGs, as they require global data.

Table 5. Basic Metrics

\begin{tabular}{|c|c|}
\hline Level & Basic Metrics \\
\hline \hline Appli- & E2E Latency, JMX/JVM Metrics \\
cation & Flume/HBase/HDFS INFO Logs \\
\hline Virtual & VCPU, Memory, I/O Metrics \\
Machine & Network Traffic, Connections \\
\hline Dom0 \& & CPU, I/O and Memory Metrics \\
System & Paging, Context Switch Metrics \\
\hline
\end{tabular}

Table 6. Built-in Functions

\begin{tabular}{|c|c|c|}
\hline & DPG & Algorithms \\
\hline \hline Watch & Hierarchy & $\begin{array}{c}\text { MAX/MIN/AVE, } \\
\text { Entropy, Top-K }\end{array}$ \\
\hline Scope & Centralized & Pathmap[3] \\
\hline Query & Centralized & $\begin{array}{c}\text { K-Clustering, } \\
\text { PCA }\end{array}$ \\
\hline
\end{tabular}

End-to-End Anomaly Detection. The Watch operation is implemented using a DPG with a hierarchical topology in which the leaves are all of the nodes 


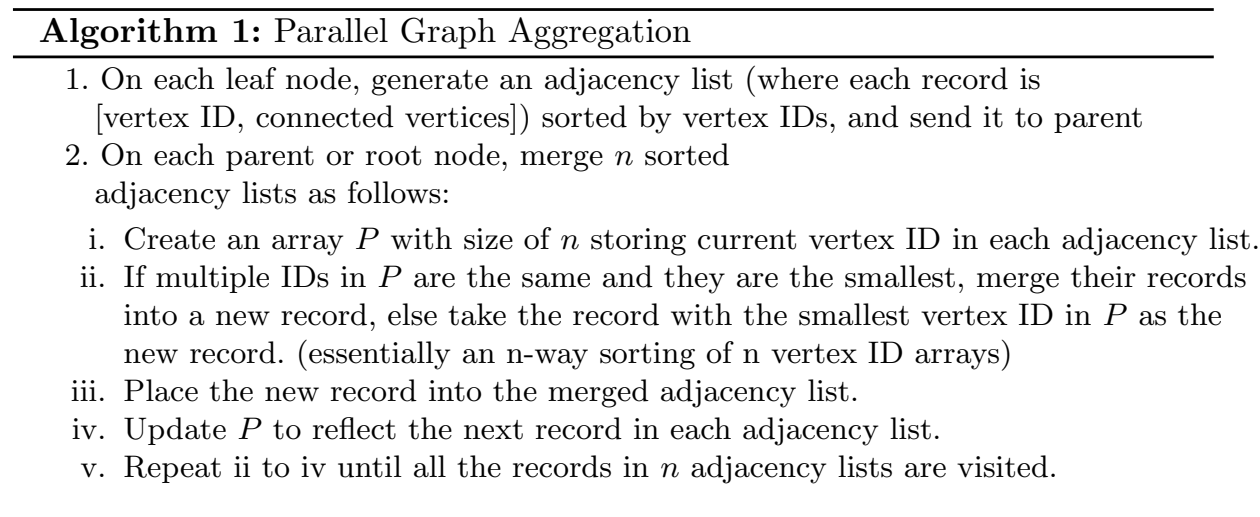

of the web log analysis application. This DPG collects the end-to-end latency on each Flume agent, which is defined as the duration between the time when a new log entry is added and the time it (or its associated result) appears in HBase. This is measured by creating a test log entry on each agent, querying the entry in HBase, and computing the difference. The latencies are then aggregated through the tree using Entropy-based Anomaly Testing (EbAT) [37,35], a lightweight anomaly detection algorithm, to output the agents that are outliers. Other algorithms for anomaly detection and ranking are investigated in $[38,34]$.

Interaction Tracking. Table 7 shows the built-in global graphs supported by Scope, covering a range of interactions from event level to network and system levels. For each graph type, in our implementation, a DPG is deployed and continuously run on all the nodes to construct and update the corresponding graph structure in VMaster. There are two ways to track the global interactions, centralized or distributed.

Table 7. VScope Interaction Graphs

\begin{tabular}{|c|c|c|}
\hline & Interaction & DPG \\
\hline \hline Causality & Event Flow & Centralized \\
Graph & between Nodes & Using Pathmap \\
\hline $\begin{array}{c}\text { Connection } \\
\text { Graph }\end{array}$ & Network & Distributed \\
Connection & Using Netstat \\
\hline Virtual & Dom0-DomU & Distributed \\
Graph & Mapping & Using Libvirt \\
\hline Tier & Dependency & Distributed \\
Graph & between Tiers & Static Config. \\
\hline
\end{tabular}

For interactions like the causality graph implemented using Pathmap [3], a DPG collects metrics from leaves, compresses them at intermediate nodes, and then constructs the graph at the DPG root. An alternate distributed implementation of graph construction uses parallel analysis in which the leaves analyze metrics to generate a local graph (e.g., in the connection graph, it is the ingress and egress connections on a node), the local graphs are aggregated at parent nodes to create partial graphs which are finally aggregated at the root to produce the global graph. The current prototype uses adjacency lists to represent graphs and employs the parallel algorithm shown in Algorithm 1 to merge adjacency lists. 


\section{Experimental Evaluation}

Experiments are conducted on a testbed running 1200 Xen VMs hosted by 60 physical server blades using Ubuntu Linux (20 VMs per server). Every server has a 1TB SATA disk, 48GB Memory, and 16 CPUs (2.40GHz). Each VM has $2 \mathrm{~GB}$ memory and at least $10 \mathrm{G}$ disk space.

\subsection{VScope Base Overheads}

We install VScope on every VM in a host and vary the number of VNodes on each VM. Each VNode collects the metrics shown in Table 5, and sends them to a separate DPG. As shown in Table 8, CPU and Memory overheads to the VM are negligible even when there are 50 VNodes (1000

Table 8. VScope Runtime Overheads

\begin{tabular}{|c|c|c|c|}
\hline $\begin{array}{c}\text { DPG\# } \\
\text { in Host }\end{array}$ & $\begin{array}{c}\text { VNode\# } \\
\text { in VM }\end{array}$ & $\begin{array}{c}\text { CPU Usage } \\
\text { Increase }\end{array}$ & $\begin{array}{c}\text { Memory Usage } \\
\text { Increase }\end{array}$ \\
\hline \hline 20 & 1 & $<0.01 \%$ & $0.02 \%$ \\
\hline 100 & 5 & $<0.01 \%$ & $0.02 \%$ \\
\hline 1000 & 50 & $<0.01 \%$ & $0.03 \%$ \\
\hline
\end{tabular}
concurrent DPGs in the host). With continuous anomaly detection and via interaction tracking, VScope imposes only $0.4 \%$ overhead on the end-to-end latency of application described in Section 4. In contrast and as shown in Section 4, heavyweight VScope operations, like those performing tracing or logging may incur considerable overheads, due to the innately high costs of those data collection methods. These facts demonstrate the utility of continuously using the 'thin' VScope layer, which does not add notable costs, and then, only using heavier weight data collection and analysis methods when needed. Further, by having the 'thin' layer point out 'where' and 'when' such heavier weight methods are to be used, the inevitably high overheads of using those methods can be reduced.

\subsection{DPG Deployment}

Fast deployment of DPGs is critical for timely troubleshooting. We evaluate this by measuring the latency for deploying a hierarchical DPG on more than 1000 VMs, each of which has one VNode. The topology has a height of 2, and the total number of leaf VMs varies from 125 to 1000.

As expected, Figure 5(a) shows increased deployment times (presented as latency on the Y-Axis) with increased DPG sizes. However, latency remains within 5 seconds even at the scale of $1000 \mathrm{VMs}$. This would be considered sufficient for current troubleshooting delay requirements stated in [8] (typically 1 hour), but it also suggests the utility of future work on DPG reuse - to use and reconfigure an existing DPG, when possible, rather than creating a new one, or to pre-deploy DPGs where they might be needed. Deploying moderate scale DPGs with hundreds of nodes, however, usually happens within 1 second, suggesting that such optimizations are not needed at smaller scale. Also note that deployment latency varies with different branching factors (bf). At scales less than 750, deploying the DPG with bf 125 has larger latency than those with smaller bf values; this is because parent nodes construct their subtrees in parallel and the parents in the DPG with bf 125 have the biggest subtrees. 


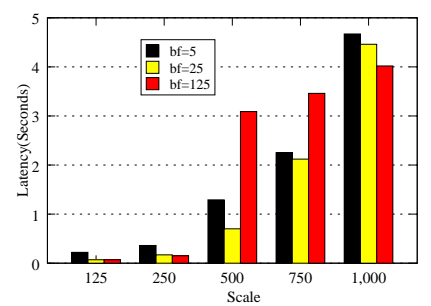

(a) DPG Deployment Time

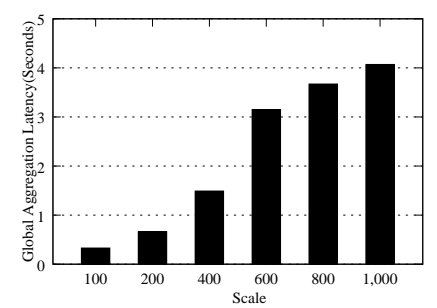

(b) Global Tracking Time

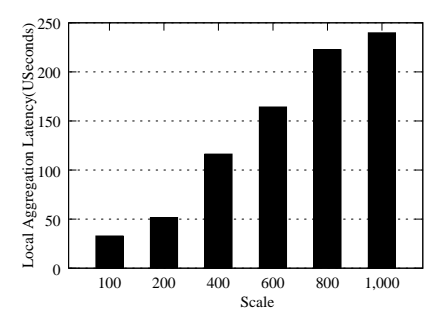

(c) Local Merge Time

Fig. 5. Efficient DPG Deployment and Guidance Mechanism

\subsection{Interaction Tracking}

The Scope operation relies on efficient methods for interaction tracking. We evaluate a distributed DPG (used for connection graph) by creating a two-level, hierarchical DPG with bf 25 . We vary its number of leaves from 125 to 1000 , and for this test, each VM has a randomly generated local interaction graph represented by an adjacency list with 1000 vertex entries with each vertex connected to 1000 randomly generated vertices. We measure the total latency from the time the first local graph is generated by leaf VMs to the time when the respective merged graph is created at the root. We also measure the average time of local processing incurred during the per-node aggregation of connection graph information in order to study the dominant factor in total latency.

As shown in Figure 5(b), the total latency for generating a global graph increases as the system scales, but it remains within 4 seconds for $1000 \mathrm{VMs}$, where each VM has a $1000 \times 1000$ local connection graph. This means that the system can generate such a global graph at a resolution of every 4 seconds. Total latency is mainly due to the queuing and dequeuing time on VNodes plus network communication time. This is shown by the small measured local aggregation latency in Figure 5(c). At the same time, since these latencies increase linearly with the total number of inputs, parallel aggregation is a useful attribute to maintain for large scale systems. We also note that the local graphs occupy a fair amount of memory, which suggests opportunities for additional optimizations through use of more efficient internal data structures. Finally, the analytics actions taken by Scope utilize the Pathmap for centralized interaction tracking. In Section 3.4, Figure 6 shows that it can generate a 1000 VM graph within 8 seconds.

In summary, the Scope operation's current implementation is efficient for the long running enterprise codes targeted in our work, but it may not meet the requirements of real-time codes such as those performing on-line sensing and actuation in highly interactive settings like immersive games.

\subsection{Supporting Diverse Analytics}

We use the algorithms in Table 6 as micro-benchmarks to measure the base performance of VScope operations. Tests randomly generate a $1000 \times 1000$ matrix of float numbers on each VM, and vary the size of the hierarchical DPG $(b f=25)$ from 125 to 1000 leaf VMs. We measure the latency for analyzing the data on 


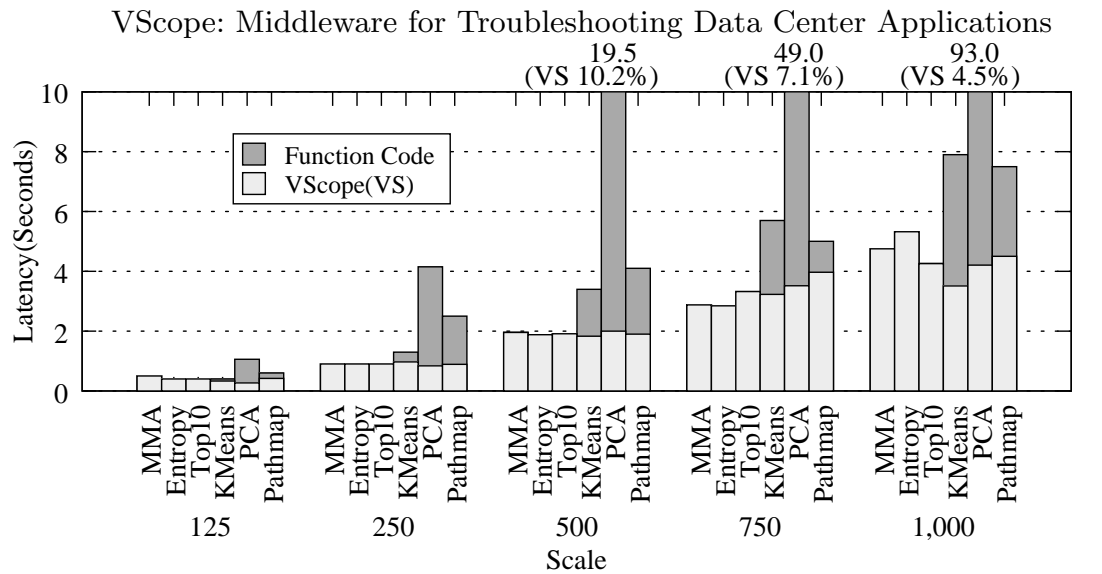

Fig. 6. Analytics Microbenchmark Performance

all leaf VMs at each scale. For centralized algorithms, the parent VNodes only relay the data. For the Top-K algorithm, we calculate the top 10 numbers. We conduct K-Means clustering with 5 passes.

Figure 6 shows latency breakdowns as well as the total latency of each function. In general, most of the algorithms operate within seconds, with increasing latencies for rising scales. Algorithms with high computational complexity are more costly, of course, but for such 'heavyweight' algorithms, especially for PCA, although the total latencies are over 1.5 minutes at the scale of $1000 \mathrm{VMs}$, the base VScope implementation contributes only about $4.5 \%$ to these delays, and this contribution decreases as the system scales.

\section{Experiences with Using VScope}

This section illustrates the utility of VScope for troubleshooting, using the application described in Figure 1 (VScope's DPG architecture was also investigated in other use cases in $[36,19]$.) The application's Flume tier has 10 collectors, each of which is linked with 20 agents. The HBase tier has 20 region servers, and the HDFS tier has 40 datanodes $^{3}$. Experiments use web request traces from the World Cup website [18] to build a log generator that replays the Apache access logs on each of 200 agent VMs. Each agent reads the new entries of the log and sends them to its collector. The collector combines the ClientID and ObjectID as the keyword and the log content as the value, then places the record into HBase. The log generator generates 200 entries per second. The worst case end-to-end latency in the problem-free scenario is within 300 milliseconds.

The VScope runtime is installed on all of the VMs and in addition, on all physical machines (i.e., Xen's Dom0s). In accordance with standard practice for management infrastructures $[21,36]$, one additional dedicated VM serves as VMaster, and 5 dedicated VMs serve as parent VNodes in the two-level hierarchy DPGs used for troubleshooting. Two use cases presented below validate VScope's utility for efficient troubleshooting.

\footnotetext{
${ }^{3}$ Each tier has one master node, and in HBase, 5 region servers serve as the ZooKeeper quorum. For simplicity, we do not 'count' masters when discussing scale.
} 


\subsection{Finding Culprit Region Servers}

The first VScope use case crosses multiple tiers of the Flume application. The objective is to find some 'culprit' region server exhibiting prolonged execution times. Those are difficult to detect with standard HBase instrumentation because debug-level logging in region servers to trace their request processing times [6] generates voluminous logs and high levels of perturbation to the running server(s). Hence troubleshooting using brute force methods might quickly find a culprit by turning on all of the region servers' debug-level logging and then analyzing these logs (in some central place), but this would severely perturb the running application. Alternative methods that successively sample some random set of servers until a culprit is found would reduce perturbation but would likely experience large delays in finding the culprit server. More generally, for multi-tier web applications, while bottleneck problems like the 'culprit' region server described above commonly occur, they are also hard to detect, for several reasons. (1) Dynamic connectivity - the connections between the Flume and HBase tiers can change, since the region server to which a collector connects is determined by the keyword region of the collector's current log entry. (2) Data-Driven concurrency - HBase splits the regions on overloaded region servers, causing additional dynamic behavior. (3) Redundancy - a region server is typically connected by multiple collectors. As a result, one 'culprit' region server exhibiting prolonged processing times may affect the end-to-end latencies observed on many agents.

We synthetically induce server slowdown, by starting garbage collection (GC) in the Java Virtual Machine (JVM) on one of the region servers. This prolonged disturbance eventually slows down the Flume agents connected to the region server via their collectors. Experimental evaluations compare VScope, the brute-force, and the sampling-based approaches for finding the culprit region server. The VScope approach follows the 3 steps illustrated in Figure 7. (1) A user at a VShell console issues a Watch operation to find which agents have prolonged endto-end latencies.

(2) Use the connection graph (chosen from Table 7) and the Scope operation to find the connected collectors and the region servers to which they connect. In these guidance actions, the connection graph is the graph parameter, the problematic

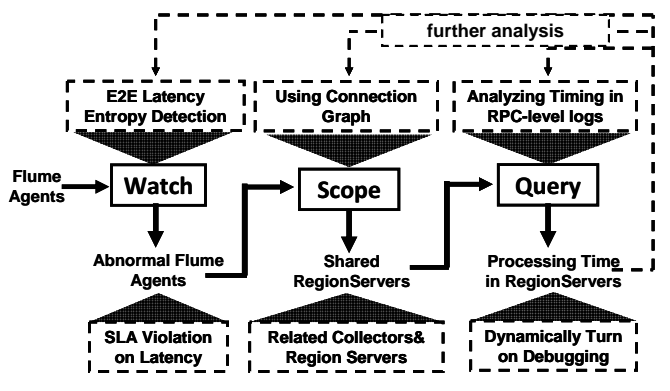

Fig. 7. Steps using VScope operations agent node is the source, and '2' is used as the distance parameter. The output will be the collector and associated region servers. By iteratively 'Scoping' all anomalous agents, we find that they share 5 collectors. Furthermore, the Scope operation returns the set of region servers in use by these collectors, and we can determine that they have 4 region servers in common. Therefore, we select those four as candidate culprits. Under the assumption of only one culprit region server, this operation will succeed because the culprit affects all of these collec- 
tors. While it will be rare to have multiple culprit region servers in a short period of time, in that case, more candidates may be chosen, but they still constitute only a small set of all region servers.

(3) Here, VScope has narrowed down the search for the problematic region server, and we can now use the Query operation to turn on debug-level logging for the candidates. We note that the region servers yielded by the Scope operation will always include the culprit, because VScope tracks all connections. The user will still have to carefully examine the region server logs to find the problem, but instead of having 20 candidates (the brute-force approach), there are just 4 . If the examination is done sequentially (by gathering and examining logs one server at a time) to minimize perturbation, the user can expect to examine 2 logs on the average (requiring 20 minutes of logging and .45GB of data) with VScope, as opposed to $10 \operatorname{logs}$ (requiring 100 minutes of logging and $2 \mathrm{~GB}$ of data) with the bruteforce approach. If $\log$ gathering is performed in parallel to save time, the information provided by VScope allows the

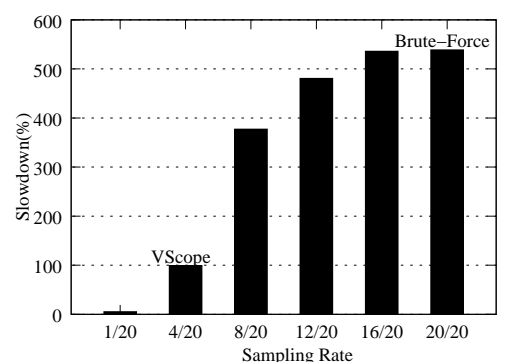

Fig. 8. E2E Performance Slowdown (i.e. E2E latency increase) \% w.r.t Sampling Rate (\# of sampled region servers / total \# of region servers) retrieval of just $4 \operatorname{logs}(0.9 \mathrm{~GB})$ vs. $20 \operatorname{logs}(4.1 \mathrm{~GB})$ by the brute-force approach. Note that, as shown in Figure 8, simultaneously logging on multiple region servers has a non-linear effect upon system performance. Simultaneous logging on only 4 servers (with VScope) slows the overall system down by $99.3 \%$, but logging on all servers (brute-force) slows it by $538.9 \%$. Compromise approaches like random sampling might log on more than one, but fewer than the total number of candidate region servers, hoping to trade off perturbation with 'time-toproblem-discovery'. However, the inherent randomness makes their performance nondeterministic. In contrast, VScope rationally narrows the set of possible bad region servers, thus improving the expected perturbation, log data sizes, and time to resolution in both average and worst cases.

These results validate the importance of VScope's 'guided' operation that explicitly identifies the nodes on which troubleshooting should focus, in contrast to methods that use sampling without application knowledge or that employ non-scalable exhaustive solutions. They also demonstrate VScope's ability to assist with cross-tier troubleshooting. We note that, for sake of simplicity, this use case assumes the root cause to be within the region servers. This assumption can be removed, of course, and in that case, operators can apply further analysis as shown in Figure 7 by iteratively using VScope operations.

\subsection{Finding a 'Naughty' VM}

Previous research has shown the potential for running real-time application in virtualized settings [22]. However, VMs' resource contention on I/O devices can degrade the end-to-end performance of the application. A typical scenario is 
that some 'naughty' VM excessively uses a physical NIC shared by other VMs on the same physical host, thereby affecting the performance of the real-time VMs. Potential 'naughty' VMs could be those that run MapReduce reducers and exchange voluminous data with a number of other nodes (e.g. mappers), or those running HDFS datanodes and replicating large files. Contention could also stem from management operations like VM migration and patch maintenance [32].

There are remedies for contention issues like those above. They include migrating the 'naughty' VMs and/or changing network scheduling. VM migration can involve long delays, and changes to VMs' network scheduling may involve kernel reboots that are unsuitable for responsive management. The solution with which we experiment performs traffic shaping for the 'naughty' VM on-the-fly, in the hypervisor, without involving guest VMs. To do so, however, support is needed to first locate the troublesome VM. VScope running in the Dom0s of our virtualized infrastructure provides such support. Specifically, VScope deploys VNodes in each host's Dom0, using the virtualization graph in Table 7 to track mappings between VMs and hypervisors.

We emulate the 'naughty' $\mathrm{VM}$ issue by deploying a VM with a Hadoop datanode and tasktracker, on the host where a 'good' VM is running one of the 200 Flume agents. This scenario is chosen to emulate co-running a real-time web $\log$ analysis application with a batch system using Hadoop for long term
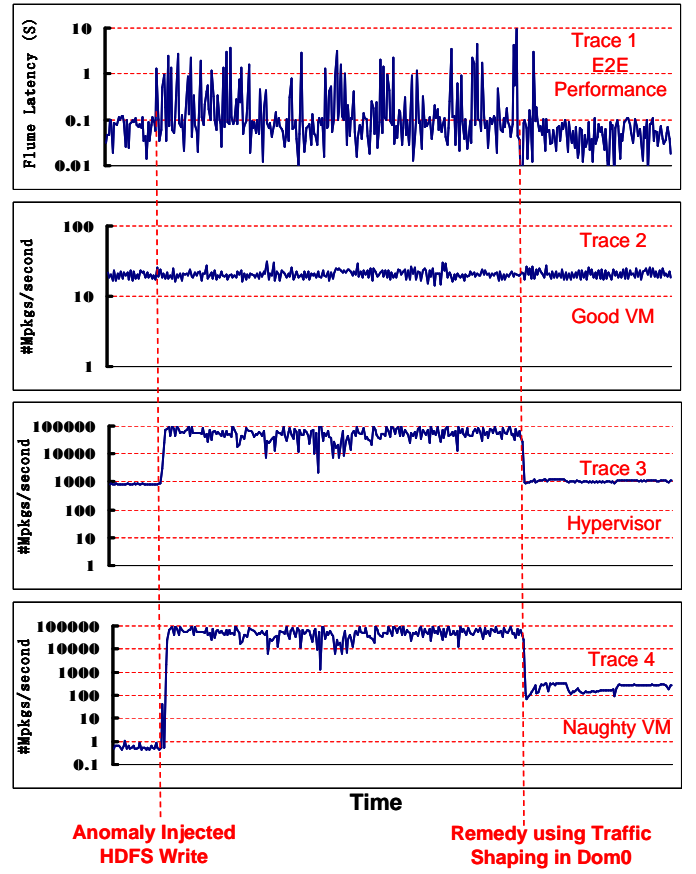

Fig. 9. Using VScope to Find a 'Naughty' VM analysis on the data generated by the real-time application. In this case, a problem is created by starting a HDFS benchmarking job called 'TestDFSIO write', which generates 120 2GB files with 4 replicas for each file in HDFS. This 'naughty VM' generates 3 files (we have 40 slaves in the Hadoop configuration. Every slave carries out 3 map tasks, each of which writes a $2 \mathrm{G}$ file to HDFS, and replicates them via the network. VScope is used to find that naughty VM, so that its communications can be regularized via Dom0 traffic shaping.

The monitoring traces in Figure 9 demonstrate VScope's troubleshooting process. Trace 1 presents the latency data generated by the Watch operation. Latency rises after the anomaly is injected. Using 1 second as the threshold for an end-to-end performance violation, after 20 violations are observed within 5 minutes, the Watch operation reports an anomaly and its location, i.e., the 
'good' VM. After the anomaly is reported, troubleshooting starts for the VM by querying basic VM level metrics, including the number of packages per second represented by Trace $2^{4}$, where we find that metrics in the VM do not show abnormal behavior. In response, we use the Scope operation to find which physical machine is hosting the VM and then Query its aggregate packet rate. With these guided actions, Trace 3 shows that the shared NIC is exchanging a large number of packets, in contradiction to the low packet rate in the 'good' VM. The next step is to further Scope the virtualization graph to find the other VMs running on the same physical host and then Query the network metrics of their VIFs ${ }^{5}$. The 'naughty' VM is easily found, because its respective VIF consumes the majority of the packets for the physical NIC, as shown in Figure 9:Trace 4. The correctness of the diagnosis obtained via VScope is demonstrated by applying traffic shaping in Dom0, which involves using TC to throttle the bandwidth of the 'naughty' VM. It is apparent that this action causes the end-to-end latency of the good VM to return to normal (see Trace 1). In Trace 3, the hypervisor packet rate goes down, and in Trace 4 the network consumption of the 'naughty' VM also sinks, as expected, but it still has its share of network bandwidth.

\section{Related Work}

Aggregation systems like SDIMS[39] and Moara[20] are most related to VScope in terms of flexibility. SDIMS provides a flexible API to control the propagation of reads and writes to accommodate different applications and their data attributes. Moara queries sub-groups of machines rather than the entire system. In both systems, flexibility is based on dynamic aggregation trees using DHTs (Distributed Hash Tables). VScope's approach differs in several ways. First, VScope can control which nodes and what metrics to analyze; neither SDIMs nor Moara provides this level of granularity. SDIMS only controls the level of propagation along the tree, and Moara chooses groups based on attributes in the query (e.g., CPU utilization). Second, the analysis functions in SDIMS and Moara are limited to aggregation functions, while arbitrary functions can be used with VScope, including those performing 'in transit' analysis. Third, like other monitoring or aggregation systems, including Ganglia[26], Astrolabe[33], and Nagios[27], SDIMS and Moara focus on monitoring the summary of system state, while VScope's can also be used for in-depth troubleshooting, including debugging and tracing, supported by basic metric aggregation like that performed in the Watch operation.

GWP[30], Dapper[31], Fay[14], Chopstix[7] are distributed tracing systems for large scale data centers. VScope is similar in that it can monitor and analyze in-depth system or application behaviors, but it differs as follows. First, instead of using statistical (Fay and Chopstix leverage sketch, a probabilistic data structure for metric collection) or random/aggressive sampling (as used in

\footnotetext{
${ }^{4}$ We only show NIC-related metrics for succinctness.

${ }^{5} \mathrm{~A}$ VIF is the logical network interface in Dom0 accepting the packets for one VM and in our configuration, each VM has a unique VIF.
} 
GWP and Dapper), VScope can look at any set of nodes, making it possible to implement a wide range of tracing strategies (including sampling) through its guidance mechanism. Second, those tracing systems use off-line analysis, while VScope can analyze data on-line and in memory, to meet the latency restriction for troubleshooting real-time applications.

HiTune[12] and $\mathrm{G}^{2}[17]$ share similarity with VScope in that they are general systems for troubleshooting 'big-data' applications. HiTune extracts the dataflows of applications, using Chukwa for data collection and Hadoop for dataflow analysis. $\mathrm{G}^{2}$ is a graph processing system that uses code instrumentation to extract runtime information as a graph and a distributed batch processing engine for processing the queries on the graph. VScope differs in its focus on on-line troubleshooting, whereas HiTune and $\mathrm{G}^{2}$ are mainly for off-line problem diagnosis and profiling. Further, HiTune and $\mathrm{G}^{2}$ are concerned with analyzing within a single application tier, while VScope troubleshoots across multiple application tiers. Other troubleshooting algorithms and systems, such as Pinpoint[10], Project5[4], and E2EProf[3], target traditional web applications while VScope focuses on real-time data-intensive applications.

\section{Conclusions}

VScope is a flexible, agile monitoring and analysis system for troubleshooting real-time multi-tier applications. Its dynamically created DPG processing overlays combine the capture of monitoring metrics with their on-line processing, (i) for responsive, low overhead problem detection and tracking, and (ii) to guide heavier weight diagnosis entailing detailed querying of potential problem sources. With 'guidance' reducing the costs of diagnosis, VScope can operate efficiently at the scales of typical data center applications and at the speeds commensurate with those applications' timescales of problem development. The paper provides evidence of this fact with a real-time, multi-tier web log analysis application.

Our ongoing work is further developing VScope's notion of guided operation, one idea being to automatically generate certain sequences of guidance actions from the previous manual actions taken by operators. We will also investigate other guidance options. To extend scalability to the 10,000+ machines of today's large scale data center applications run by web companies like Google or Amazon, it may also be useful to pre-position DPGs into potentially critical subsystems and/or reconfigure existing DPGs, instead of deploying new ones when investigating problems via detailed queries.

\section{References}

1. The evpath library. http://www.cc.gatech.edu/systems/projects/EVPath.

2. D. Abadi, D. Carney, U. Çetintemel, M. Cherniack, C. Convey, S. Lee, M. Stonebraker, N. Tatbul, and S. Zdonik. Aurora: a new model and architecture for data stream management. The VLDB Journal, 12(2):120-139, 2003.

3. S. Agarwala, F. Alegre, K. Schwan, and J. Mehalingham. E2eprof: Automated end-to-end performance management for enterprise systems. In Proceedings of the 37th Annual IEEE/IFIP International Conference on Dependable Systems and Networks, DSN '07, pages 749-758, Washington, DC, USA, 2007. IEEE. 
4. M. K. Aguilera, J. C. Mogul, J. L. Wiener, P. Reynolds, and A. Muthitacharoen. Performance debugging for distributed systems of black boxes. In Proceedings of the 19th ACM symposium on Operating systems principles, SOSP '03, 2003.

5. Apache. Cloudera flume. http://archive.cloudera.com/cdh/3/flume/.

6. Apache. Hbase log. http://hbase.apache.org/book/trouble.log.html.

7. S. Bhatia, A. Kumar, M. E. Fiuczynski, and L. Peterson. Lightweight, highresolution monitoring for troubleshooting production systems. In Proceedings of the 8th USENIX conference on Operating systems design and implementation, OSDI'08, pages 103-116, Berkeley, CA, USA, 2008. USENIX Association.

8. P. Bodik, M. Goldszmidt, A. Fox, D. B. Woodard, and H. Andersen. Fingerprinting the datacenter: automated classification of performance crises. In Proceedings of the 5th European conference on Computer systems, EuroSys '10, pages 111-124, New York, NY, USA, 2010. ACM.

9. G. Candea, S. Kawamoto, Y. Fujiki, G. Friedman, and A. Fox. Microreboot - a technique for cheap recovery. In Proceedings of the 6th Conference on Symposium on Opearting Systems Design \& Implementation, OSDI'04, 2004.

10. M. Y. Chen, E. Kiciman, E. Fratkin, A. Fox, and E. Brewer. Pinpoint: Problem determination in large, dynamic internet services. In Proceedings of the 2002 International Conference on Dependable Systems and Networks, DSN '02, pages 595-604, Washington, DC, USA, 2002. IEEE Computer Society.

11. T. Condie, N. Conway, P. Alvaro, J. M. Hellerstein, K. Elmeleegy, and R. Sears. Mapreduce online. In Proceedings of the 7th USENIX conference on Networked systems design and implementation, NSDI'10, 2010.

12. J. Dai, J. Huang, S. Huang, B. Huang, and Y. Liu. Hitune: dataflow-based performance analysis for big data cloud. In Proceedings of the 2011 USENIX conference on USENIX annual technical conference, USENIXATC'11, 2011.

13. M. De Hoon, S. Imoto, J. Nolan, and S. Miyano. Open source clustering software. Bioinformatics, 20(9):1453-1454, June 2004.

14. U. Erlingsson, M. Peinado, S. Peter, and M. Budiu. Fay: extensible distributed tracing from kernels to clusters. In Proceedings of the Twenty-Third ACM Symposium on Operating Systems Principles, SOSP '11, pages 311-326, 2011.

15. Facebook. Scribe. https://github.com/facebook/scribe/wiki.

16. B. Gedik, H. Andrade, K.-L. Wu, P. S. Yu, and M. Doo. Spade: the system s declarative stream processing engine. In Proceedings of the 2008 ACM SIGMOD international conference on Management of data, SIGMOD '08, pages 1123-1134.

17. Z. Guo, D. Zhou, H. Lin, M. Yang, F. Long, C. Deng, C. Liu, and L. Zhou. $g^{2}$ : a graph processing system for diagnosing distributed systems. In Proceedings of the 2011 USENIX annual technical conference, USENIXATC'11, 2011.

18. Hewlett-Packard. Worldcup98 logs. http://ita.ee.lbl.gov/.

19. L. Hu, K. Schwan, A. Gulati, J. Zhang, and C. Wang. Net-cohort: Detecting and managing vm ensembles in virtualized data centers. In Proceedings of the 9th ACM International Conference on Autonomic Computing, ICAC '12, 2012.

20. S. Y. Ko, P. Yalagandula, I. Gupta, V. Talwar, D. Milojicic, and S. Iyer. Moara: flexible and scalable group-based querying system. In Proceedings of the 9th ACM/IFIP/USENIX International Conference on Middleware, Middleware '08, pages 408-428, New York, NY, USA, 2008. Springer-Verlag New York, Inc.

21. S. Kumar, V. Talwar, V. Kumar, P. Ranganathan, and K. Schwan. vmanage: loosely coupled platform and virtualization management in data centers. In Proceedings of the 6th international conference on Autonomic computing, ICAC '09, pages 127-136, New York, NY, USA, 2009. ACM. 
22. M. Lee, A. S. Krishnakumar, P. Krishnan, N. Singh, and S. Yajnik. Supporting soft real-time tasks in the xen hypervisor. In Proceedings of the 6th ACM SIGPLAN/SIGOPS international conference on Virtual execution environments, VEE '10, pages 97-108, New York, NY, USA, 2010. ACM.

23. LinkedIn. Kafka. http://sna-projects.com/kafka/design.php.

24. M. Mansour and K. Schwan. I-rmi: performance isolation in information flow applications. In Proceedings of the ACM/IFIP/USENIX 2005 International Conference on Middleware, Middleware '05, pages 375-389, 2005.

25. N. Marz. Twitter's storm. https://github.com/nathanmarz/storm.

26. M. L. Massie, B. N. Chun, and D. E. Culler. The ganglia distributed monitoring system: Design, implementation and experience. Parallel Computing, 2003.

27. L. Nagios Enterprises. Nagios. http://www.nagios.org/documentation.

28. L. Neumeyer, B. Robbins, A. Nair, and A. Kesari. S4: Distributed stream computing platform. In Data Mining Workshops (ICDMW), 2010 IEEE International Conference on, pages $170-177$, dec. 2010.

29. A. Rabkin and R. Katz. Chukwa: a system for reliable large-scale log collection. In Proceedings of the 24th international conference on Large installation system administration, LISA'10, pages 1-15, Berkeley, CA, USA, 2010.

30. G. Ren, E. Tune, T. Moseley, Y. Shi, S. Rus, and R. Hundt. Google-wide profiling: A continuous profiling infrastructure for data centers. Micro, IEEE, 2010.

31. B. H. Sigelman, L. A. Barroso, M. Burrows, P. Stephenson, M. Plakal, D. Beaver, S. Jaspan, and C. Shanbhag. Dapper, a large-scale distributed systems tracing infrastructure. Technical Report dapper-2010-1, Google, Apr 2010.

32. V. Soundararajan and J. M. Anderson. The impact of management operations on the virtualized datacenter. In Proceedings of the 37th annual international symposium on Computer architecture, ISCA '10, pages 326-337, 2010.

33. R. Van Renesse, K. P. Birman, and W. Vogels. Astrolabe: A robust and scalable technology for distributed system monitoring, management, and data mining. $A C M$ Trans. Comput. Syst., 21:164-206, May 2003.

34. K. Viswanathan, L. Choudur, V. Talwar, C. Wang, G. MacDonald, and W. Satterfield. Ranking anomalies in data centers. In the 13th IEEE/IFIP Network Operations and Management Symposium, NOMS'12, pages 79-87, 2012.

35. C. Wang. Ebat: online methods for detecting utility cloud anomalies. In Proceedings of the 6th Middleware Doctoral Symposium, MDS '09, 2009.

36. C. Wang, K. Schwan, V. Talwar, G. Eisenhauer, L. Hu, and M. Wolf. A flexible architecture integrating monitoring and analytics for managing large-scale data centers. In Proceedings of the 8th ACM international conference on Autonomic computing, ICAC '11, pages 141-150, New York, NY, USA, 2011. ACM.

37. C. Wang, V. Talwar, K. Schwan, and P. Ranganathan. Online detection of utility cloud anomalies using metric distributions. In the 12th IEEE/IFIP Network Operations and Management Symposium, NOMS'10, pages 96-103, 2010.

38. C. Wang, K. Viswanathan, L. Choudur, V. Talwar, W. Satterfield, and K. Schwan. Statistical techniques for online anomaly detection in data centers. In the 12th IFIP/IEEE International Symposium on Integrated Network Management, IM'11, pages $385-392,2011$.

39. P. Yalagandula and M. Dahlin. A scalable distributed information management system. In Proceedings of the 2004 conference on Applications, technologies, architectures, and protocols for computer communications, SIGCOMM '04, pages 379-390, New York, NY, USA, 2004. ACM. 